\title{
Fractional exhaled nitric oxide in preterm-born subjects: A systematic review and meta-analysis
}

\author{
Christopher W. Course BSc, MBBCh, $\mathrm{MRCPCH}^{1}$ (D) । \\ Sailesh Kotecha PhD, FRCPCH ${ }^{2}$ (D) | Sarah J. Kotecha $\mathrm{PhD}^{2}$ (D)
}

${ }^{1}$ Welsh Regional Neonatal Intensive Care Unit, University Hospital of Wales, Cardiff, UK

2 Department of Child Health, School of Medicine, Cardiff University, Cardiff, UK

\section{Correspondence}

Dr. Sarah Kotecha, PhD, Department of Child Health, School of Medicine, Cardiff University, Heath Park, Cardiff CF14 4XN, UK.

Email: kotechasj@cardiff.ac.uk

Funding information

MRC, Grant number: MR/M022552/1

\begin{abstract}
Background: Decreased lung function is common in preterm-born survivors. Increased fractional exhaled nitric oxide (FeNO) appears to be a reliable test for eosinophillic airway inflammation especially in asthma. We, systematically, reviewed the literature to compare FeNO levels in preterm-born children and adults who did or did not have chronic lung disease of prematurity (CLD) in infancy with term-born controls.

Methods: We searched eight databases up to February 2018. Studies comparing FeNO levels in preterm-born subjects ( $<37$ weeks' gestation) in childhood and adulthood with and without (CLD) with term-born subjects were identified and extracted by two reviewers. Data were analysed using Review Manager v5.3.

Results: From 6042 article titles, 183 full articles were screened for inclusion. Nineteen studies met the inclusion criteria. Seventeen studies compared FeNO levels in pretermand term-born children and adults; 11 studies (preterm $n=640$ and term $n=4005$ ) were included in a meta-analysis. The mean FeNO concentration difference between the preterm-born and term-born group was $-0.74(95 \% \mathrm{Cl}-1.88$ to 0.41$) \mathrm{ppb}$. For the six studies reporting data on CLD (preterm $n=204$ and term $n=211$ ) the mean difference for FeNO levels was $-2.82(95 \% \mathrm{Cl}-5.87$ to 0.22$)$ ppb between the pretermborn CLD and term-born groups.

Conclusions: Our data suggest that preterm born children with and without CLD have similar FeNO levels to term-born children suggesting an alternative mechanism to eosinophilic inflammation for symptoms of wheezing and airway obstruction observed in preterm-born subjects.
\end{abstract}

\section{KEYWORDS}

asthma, bronchopulmonary dysplasia, chronic lung disease of prematurity, FeNO, fractional exhaled nitric oxide, prematurity

Presentation at meetings: The results were presented in a poster discussion session at the European Respiratory Society in Paris in September 2018.

This is an open access article under the terms of the Creative Commons Attribution License, which permits use, distribution and reproduction in any medium, provided the original work is properly cited.

(c) 2019 The Authors. Pediatric Pulmonology Published by Wiley Periodicals, Inc. 


\section{1 | INTRODUCTION}

Adverse events in early life and their impact on longer term health and morbidity are increasingly recognized. ${ }^{1}$ Preterm birth is a wellrecognized early adverse event, with potential lifelong health implications. Approximately $11 \%$ of all infants are born prematurely world-wide with rates increasing in many countries. ${ }^{2}$ Prediction and prevention of preterm birth is currently limited thus characterizing the long-term effects of preterm birth is vital to optimize their future quality of life.

The lungs are one of the last major body system to mature in utero, therefore, preterm infants are born with immature airway structures with decreased gas exchange surface. ${ }^{3}$ Respiratory pathology is the commonest consequence of preterm birth, manifesting as neonatal respiratory distress syndrome, a consequence of structurally immature lungs and pulmonary surfactant deficiency. These infants often require ventilatory support, both invasive and non-invasive, and supplementary oxygen. A subset of these infants will go on to develop chronic lung disease of prematurity (CLD), ${ }^{4}$ often defined as supplementary oxygen requirement at 36 weeks' corrected gestational age. ${ }^{5}$

Nitric oxide (NO) has several functions in the lung including neurotransmission, vasodilatation, bronchial dilatation, and immune enhancement. However, in diseases such as asthma, its actions can be paradoxical. Animal studies have shown that at lower concentrations, NO has a bronchodilator effect, but at higher levels NO has a proinflammatory action. The increased levels of NO observed in disease states caused by airway inflammation are thought to be the result of increased induction of nitric oxide synthease and of increased reactive nitrosylation pathways. ${ }^{6}$

Fractional exhaled nitic oxide (FeNO) has been demonstrated to be a useful biomarker of eosinophilic airway inflammation. FeNO levels can be easily measured non-invasively by a single-breath exhalation technique and, through utilizing mathematical airway modelling, is thought to provide a representation of distal airway inflammation. ${ }^{6}$ Although commonly thought of as an eosinophilic disease, airway inflammation in asthma can also be neutrophilic in origin. ${ }^{7}$ FeNO levels have been shown to be higher in children with asthma and during asthma exacerbations, being associated with increased airway inflammation. ${ }^{8}$ It was reported in a systematic review in 2017 that FeNO is a reasonably accurate diagnostic test for identifying subjects with asthma, with good specificity and acceptable sensitivity, especially in those with eosinophilic lung disease. FeNO is not only widely used as a diagnostic tool, but serial measurements have also been shown to be useful for monitoring treatment responses to inhaled corticosteroids. ${ }^{10}$

Several studies have shown evidence of persistent abnormal lung function in later childhood for infants diagnosed with CLD. In previous systematic reviews, we have reported that preterm-born subjects have deficits in $\mathrm{FEV}_{1}$, increased rates of bronchial hyper-responsiveness, and single doses of bronchodilators appeared to improve FEV $1 .{ }^{11-13} \mathrm{It}$ has been reported that preterm-born subjects have higher rates of wheezing and are often diagnosed with asthma. The systematic review by Been et al. reported increased rates of wheezing in preterm-born subjects. ${ }^{14}$ In addition, we have reported increased rates of wheezing in preterm-born children which was independent of a family history of atopy. ${ }^{15}$ We would question the diagnostic labelling of preterm-born subjects with the label asthma, especially as the mechanisms underlying the deficits in lung function and increased rates of bronchial hyper-responsiveness remain uncertain. We believe that the mechanisms are likely to differ to asthma thus sought if FeNO was a useful diagnostic test for preterm-born children as it is for asthma.

Thus, we conducted a systematic review and meta-analysis to identify:

a) if FeNO level is increased after preterm birth when compared to term-born controls, and

b) if FeNO level is increased in preterm-born subjects who had CLD compared to term-born subject.

\section{2 | METHODS}

We ran search strategies which included keywords relating to FeNO, preterm-birth and study design in eight databases; and additionally we searched references in the included articles to identify additional papers reporting FeNO levels in preterm-born subjects compared to term-born subjects (see Supplementary Protocol for protocol, search strategy, and data collection table). The eight databases searched were Web of Knowledge, Scopus, CINAHL, OpenSIGLE, Medline, Medline in process, EMBASE, and HMIC. Ethical approval was deemed not necessary.

\section{1 | Eligibility criteria}

Studies on FeNO levels in preterm-born children and adults subjects over 2 years of age, with or without CLD compared to term-born subjects were included. Preterm-born was defined as birth at $<37$ weeks' gestation and term-born as birth at $\geq 37$ weeks' gestation. We accepted the authors' definitions of CLD. All methods of assessing FeNO levels were accepted. However, only studies which reported the concentration of FeNO in the preterm-born and a comparator termborn groups were included. Studies in all languages from all countries were considered.

\section{2 | Study selection}

Searches were carried out in February 2018. Two reviewers (SJK and CC) independently screened each reference title and available abstracts, using the inclusion criteria in the protocol. Any reference title that met the inclusion criteria as judged by either CC or SJK was then included in the next stage where the complete full manuscript was obtained. SJK and CC then reviewed the full manuscript against the inclusion criteria. Where there was agreement, the manuscript was included in the next stage and data were extracted. Where there was 
disagreement, a third reviewer (SK) reviewed the complete manuscript and arbitrated the final decision.

\section{3 | Data collection process}

SJK data extracted data from included articles into the data tables, which were independently verified by CC. If data was not in a format which enabled the data to be included in the systematic review, the authors were contacted for further information wherever possible. Where multiple manuscripts from the same cohort were identified, they were reviewed by SJK and SK and a decision made about which article should be included, based on the largest, most complete cohort.

\section{4 | Outcome measures}

FeNO level in the preterm-born subjects and in the preterm-born subjects with CLD compared with a term control group.

\section{$2.5 \mid$ Analysis of results}

A formal meta-analysis was conducted for the studies which reported the mean and standard deviation, (or where they could be easily obtained) of FeNO (a) in the preterm (with and without CLD) and control groups, and (b) in the preterm with CLD group and term control group. The results of all the studies are also presented descriptively.

\section{6 | Statistical analysis}

Statistical analyses were performed using Review Manager (RevMan) Version 5.3. ${ }^{16}$ After initial exploration of the data, we used random effects meta-analyses to allow for heterogeneity. Medians were converted to means using the method of Wan et al. where enough information was present to allow this to be calculated. ${ }^{17} \mathrm{~A}$ calculator was used to convert $95 \%$ confidence intervals to standard deviation. ${ }^{18}$ In addition, a sensitivity analysis was conducted: studies where the means and standards deviations were calculated from medians, and the single study where standard deviations were calculated from $95 \%$ confidence intervals were removed from the meta-analysis.

\section{3 | RESULTS}

\section{1 | Studies selected and their characteristics}

A total of 6042 article titles were identified of which 183 full articles were screened for inclusion. Nineteen met the inclusion criteria (Figure 1). ${ }^{19-37}$ For the three studies that overlapped data were kindly supplied by the authors. ${ }^{32-34}$ In addition, a further author kindly supplied their data so the study could be included. ${ }^{35}$ Demographics of included articles are shown in Supplementary Table S1. Medians and IQRs or ranges were converted to means and standard deviations for three studies. ${ }^{23,25,28}$ Mean and $95 \% \mathrm{Cl}$ were converted to mean and standard deviation for one study. ${ }^{24}$

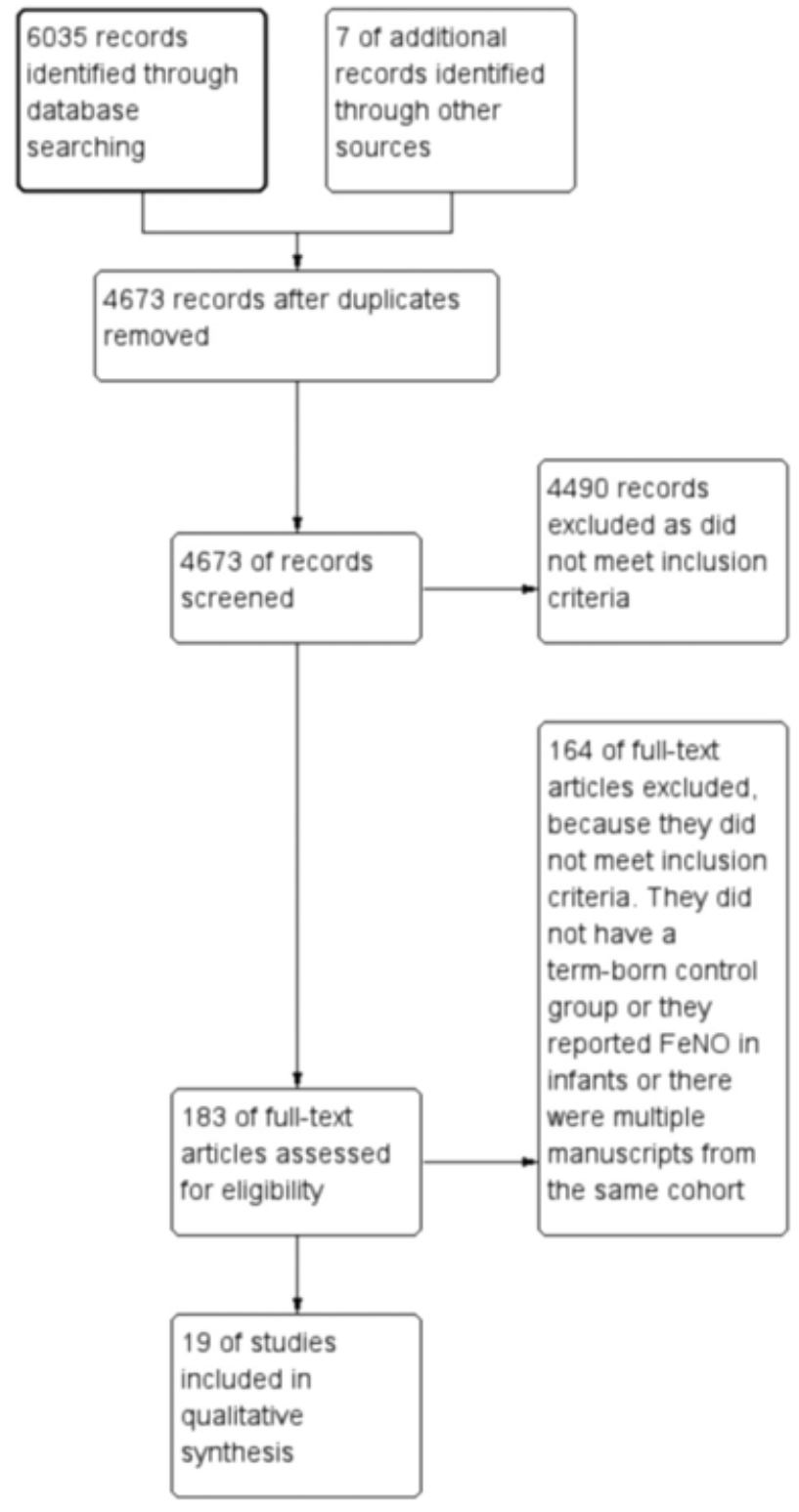

FIGURE 1 Study selection results

\section{2 | Study outcomes}

\subsubsection{FeNO in preterm group compared to term control group}

From the 17 studies identified, 11 (preterm $n=640$ and term $n=4005$ ) were included in a meta-analysis and 6 are described in detail below. The results of the meta-analysis are shown in Figure 2. The mean difference for FeNO level between the preterm-born and term-born group was $-0.74(95 \% \mathrm{Cl}-1.88$ to 0.41$) \mathrm{ppb}$. The studies are described in detail in Supplementary Table S1.

\subsubsection{FeNO in preterm group who had CLD in infancy compared to term control group}

From the 17 studies identified, 6 (preterm $n=204$ and term $n=211$ ) were included in a meta-analysis as shown in Figure 3 . The mean 
(i) WILEY

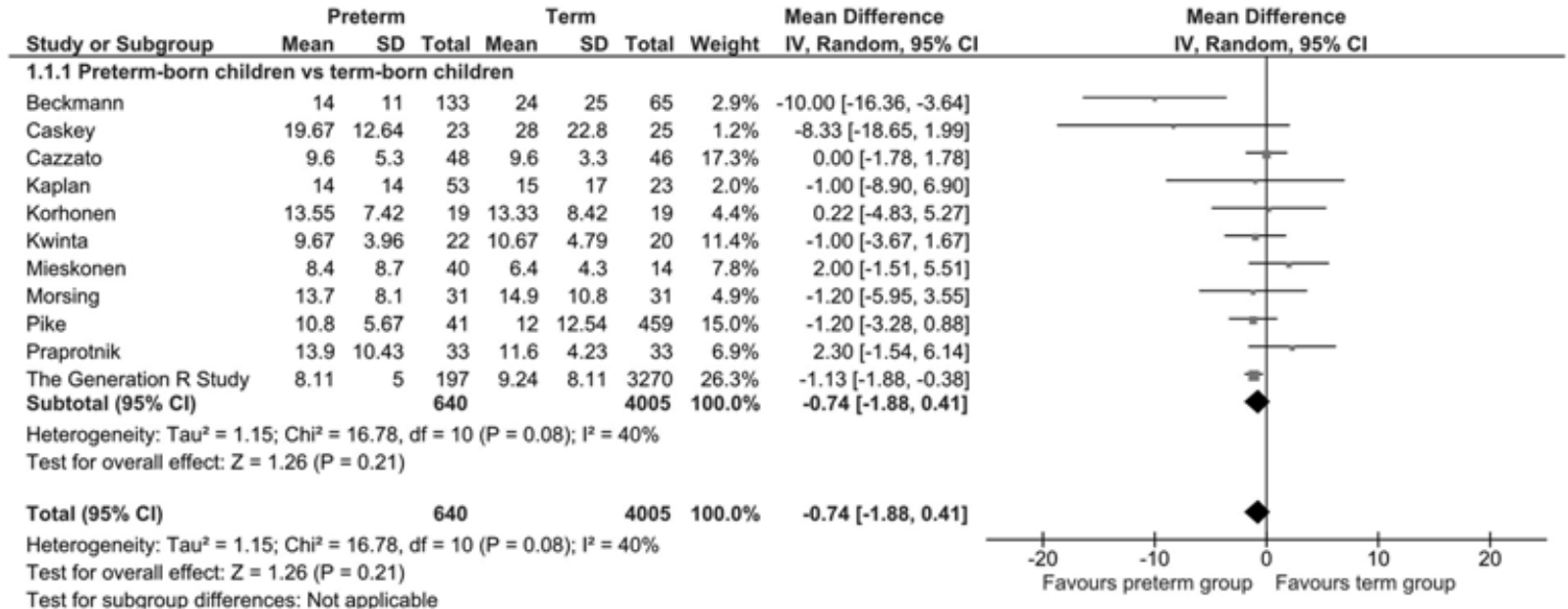

FIGURE 2 Mean FeNO of the preterm-born group compared to the term-born group

difference for FeNO levels between the preterm-born and term-born group was $-2.82(95 \% \mathrm{Cl}-5.87$ to 0.22$) \mathrm{ppb}$. The studies are described in detail in Supplementary Table S1.

\subsection{3 | Sensitivity analysis}

Removing the four studies where the means and/or standard deviations were calculated from the meta-analysis reporting the FeNO levels in the preterm group compared to the term group did not alter the mean difference substantially (mean $-0.92(95 \% \mathrm{Cl}$ -2.28 to 0.45$) \mathrm{ppb}$. Removing the three studies where the means and/or standard deviations were calculated from the meta-analysis reporting the FeNO levels in the preterm group who had CLD in infancy compared to the term group did alter the mean difference substantially $(-4.11$ (mean $95 \% \mathrm{Cl}-10.72$ to 2.50$)$ ppb. However, by removing the three studies, only three studies were left in the metaanalysis and the number of preterm-born children dropped from 204 to 144 , with the number of term-born children dropping from 211 to 134.

\subsection{4 | Description of studies not included in the meta-analyses}

Six studies were not included in a meta-analysis. They are described in detail in Supplementary Table S1 and briefly summarised below with data for FeNO given in ppb. In general, the preterm-born subjects with and without CLD compared to the term-born group had statistically similar or lower levels of FeNO compared to control groups. Most were excluded as they either reported data as geometric means or as medians.

1. Nordlund et al. ${ }^{19}$ studied 30 preterm-born children with CLD and 30 term born children with asthma sensitized to airborne allergens at a mean age of approximately 10 years and reported a significantly lower FeNO level in the preterm-born children with CLD (median FeNO in the CLD group 7.9, in the asthma group 13.3).

2. Baraldi et al. ${ }^{20}$ studied 31 preterm-born children with CLD, 31 preterm-born children without CLD, 31 term controls and 31 patients with asthma at an approximate mean age of 8 years. The two preterm-born groups had the lowest FeNO levels when

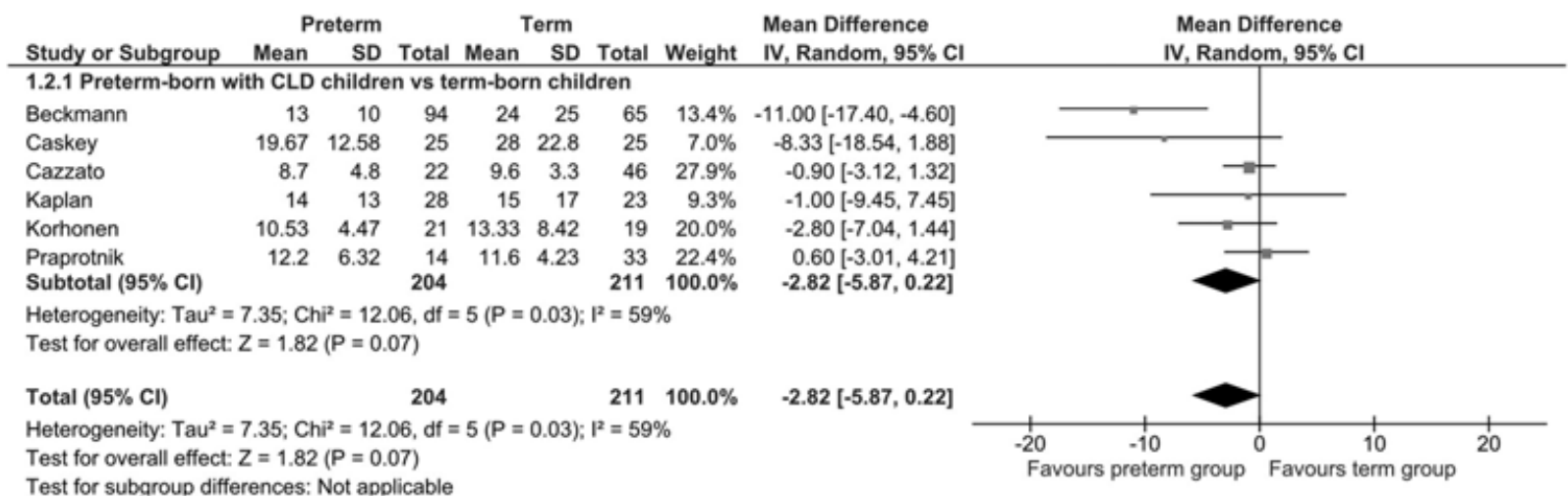

FIGURE 3 Mean FeNO of the preterm-born group with CLD compared to the term-born group 
compared to the term controls and the patients with asthma. The CLD group had statistically significantly lower levels of FeNO compared to the other three groups (geometric mean FeNO in the CLD group 7.7, term 10.7, preterm-born no CLD 9.9, asthma 24.9).

3. Kilbride at al. ${ }^{21}$ studied extremely low birth-weight preterm-born children, heavier preterms and term-born children with a normal birthweight at 12-15 years of age FeNO results did not differ by birthweight category (FeNO in ELBW preterm-born children 10.2, heavier preterm born children 15.2, and normal birthweight termborn children 10.4).

4. Vollsaeter at al. ${ }^{30}$ also studied 45 preterm-born adults, who were subdivided by CLD severity, and compared the CLD groups to 39 term-born, 18-25 year-old adults. FeNO levels did not differ between the groups (geometric mean FeNO terms 11.8, No-CLD 11.7, Mild CLD 12.2, moderate/severe CLD 11.5).

5. Vollsaeter at al. ${ }^{36}$ studied preterm-born children with and without CLD and term-born controls at a mean age of approximately 11 years. FeNO levels did not differ significantly between the groups (geometric mean FeNO terms 11.77, preterm no CLD 10.46, preterm CLD 9.08).

6. Malmberg et al. ${ }^{37}$ studied very low birthweight children, children with a history of wheeze and term-born children at 8-10 years of age. The FeNO levels were not significantly different between the groups once height was adjusted for (geometric mean FeNO terms 9.9, and VLBW 10.7).

\section{4 | DISCUSSION}

To our knowledge, this is the first systematic review and meta-analysis examining FeNO levels in preterm infants, with and without CLD, compared to infants born at term. FeNO level is generally higher in subjects with asthma, but especially in those who have eosinophilic airway inflammation. FeNO has been established as a reliable, noninvasive test for aiding the diagnosis of eosinophilic asthma, with reasonable sensitivity and good specificity. ${ }^{9}$ A recent review studying exhaled biomarkers summarized the evidence to date with the statement that "FeNO is consequently considered a marker of a common asthma endotype characterized by Th2-mediated airway inflammation, eosinophilia, and responsiveness to inhaled steroids". The results of our systematic review and meta-analyses suggest that subjects born preterm have no statistically significant difference in FeNO levels when compared to term-born subjects. Preterm-born subjects with CLD also had similar results to term-born subjects. The latter results should be treated with caution as the number of studies and total number of subjects was smaller; and the mean difference was altered by the removal of the studies where the means and/or standard deviations were calculated from medians or $95 \% \mathrm{Cls}$.

Despite improvements in neonatal management over the past 20 years, including the routine use of antenatal steroids, surfactant, and gentle mechanical ventilation modes, CLD remains a significant consequence of preterm birth at an immature stage of pulmonary development. Preterm born infants are known to experience long-term respiratory morbidity during the rest of childhood. Those with a diagnosis of CLD are most vulnerable. Preterm born infants with and without CLD are more prone to wheezing symptoms, more frequently use inhaled bronchodilator treatments, are more likely to be admitted to hospital for management of wheeze or dyspnoea, and are more than twice as likely to be labelled with a diagnosis of asthma. ${ }^{38}$ This increased respiratory morbidity appears to extend beyond childhood into adulthood. ${ }^{39}$ However, the reasons for continuing respiratory illness in childhood and beyond remains unclear.

Our results suggest that preterm-born subjects are unlikely to have eosinophilic airway inflammation underlying their respiratory symptoms and may not have the same airway mechanics as those children with a diagnosis of asthma. The data suggest that there may be alternative underlying mechanisms to explain their episodic or chronic wheezing and symptoms of airway obstruction. Whether that is structural or functional remains to be confirmed; our recent systematic review reported increased bronchial hyper-responsiveness in pretermborn subjects compared to term-born subjects by both direct and indirect methods of measuring bronchial hyper-responsiveness. When only subjects who were preterm-born and had CLD in infancy were studied, the differences were greater, suggesting greater pathology of their airways. ${ }^{13}$ We have also reported increased exercise-induced bronchial hyper-responsiveness in school-age children with a background of CLD which is responsive to bronchodilator therapy. ${ }^{40}$

With increasing number of children surviving preterm-birth, it is important to understand the impact of preterm birth at a critical stage of lung development. Currently, the mechanisms underlying the increased respiratory symptoms, deficits in lung function, increased bronchial hyper-responsiveness, responses to single doses of a bronchodilator are not fully understood. Our data adds that airway eosinophilic inflammation is unlikely since FeNO level was not increased in the preterm groups including in those with CLD when compared to their term counter-parts. However, FeNO may be affected by abnormal lung development in patients with CLD, so it will be difficult to exclude completely eosinophilic airway inflammation. We have recently shown that preterm-born subjects may have specific wheezing phenotypes. ${ }^{41}$ It may be that specific phenotypes are associated with increased FeNO. There are suggestions that neutrophilic or oxidant injury may be continuing but these small studies need to be replicated including to identify the reasons why such injury is continuing especially given that the initial injuries most likely occurred perinatally.

The authors used various different methods to measure FeNO and, importantly, used differing methods to represent their data including as means, medians or geometric means; or as proportions of children with greater than a predefined value of FeNO, most commonly $35 \mathrm{ppb}$. This led to difficulty in interpreting the data between the different studies. Thus, it is important in any future task force where expert opinion is sought to include the most appropriate way of reporting FeNO concentrations. Given that the data is unlikely to be normally distributed, geometric means together with proportions of children with greater than predefined FeNO values may be the most appropriate way forward. 


\section{5 | LIMITATIONS}

As with all systematic reviews we were limited by the quality and data supplied in the studies included in the meta-analysis. The data was not always normally distributed hence many authors did not present their results as means and standard deviations. We converted medians where possible to means and standard deviations, but this may have introduced errors. However, we carried out a sensitivity analysis to explore this. In addition, the heterogeneity of the studies included is also a limitation. There was heterogeneity due to the fact the preterm and term-born subjects were born over a number of years, were tested at a range of ages, and were born at a range of gestations, during a period where standards of neonatal intensive care and management of extreme prematurity have changed, including the introduction of antenatal steroids and surfactant. In addition, FeNO levels were not measured with same make of device in all the included studies.

\section{6 | CONCLUSION}

Our systematic review and meta-analysis showed that infants born preterm, both with and without CLD had similar FeNO values to termborn controls. Despite preterm born children being more likely to show wheezing symptoms and be labelled with a diagnosis of asthma, our data suggests that their respiratory symptoms and airway obstruction are unlikely to be mainly due to eosinophilic airway inflammation thus unlikely to benefit from the diagnostic label of asthma. Future research should aim to further the underlying mechanisms explaining the longterm respiratory morbidity observed in preterm-born children and to accurately identify biomarkers to tailor their treatments to their specific respiratory pathology.

\section{ACKNOWLEDGMENTS}

We are very grateful to the authors who supplied additional data.

\section{CONFLICT OF INTEREST}

The authors have no conflicts of interest to report.

\section{FUNDING}

SK reports funding from MRC (MR/M022552/1) for this submitted work.

\section{ORCID}

Christopher W. Course iD http://orcid.org/0000-0002-7789-2057

Sailesh Kotecha (iD http://orcid.org/0000-0003-3535-7627

Sarah J. Kotecha iD http://orcid.org/0000-0001-5640-0300

\section{REFERENCES}

1. Gluckman PD, Hanson MA, Cooper C, Thornburg KL. Effect of in utero and early-life conditions on adult health and disease. $N$ Engl $J$ Med. 2008;359: 61-73.

2. Blencowe $\mathrm{H}$, Cousens $\mathrm{S}$, Oestergaard $\mathrm{MZ}$, et al. National, regional, and worldwide estimates of preterm birth rates in the year2010 with time trends since 1990 for selected countries: a systematic analysis and implications. Lancet. 2012;379: 2162-2172.

3. Pickerd N, Kotecha S. Pathophysiology of respiratory distress syndrome. Paediatr Child Health. 2009;19: 153-157.

4. Baraldi E, Filippone M. Chronic lung disease after premature birth. N Engl J Med. 2007;357: 1946-1955.

5. Shennan AT, Dunn MS, Ohlsson A, Lennox K, Hoskins EM. Abnormal pulmonary outcomes in premature infants: prediction from oxygen requirement in the neonatal period. Pediatrics. 1988;82: 527-532.

6. Hoyte FCL, Gross LM, Katial RK. Exhaled nitric oxide: an update. Immunol Allergy Clin North Am. 2018;38: 573-585.

7. Douwes J, Gibson P, Pekkanen J, Pearce N. Non-eosinophilic asthma: importance and possible mechanisms. Thorax. 2002;57: 643-648.

8. Ferraro V, Carraro S, Bozzetto S, Zanconato S, Baraldi E. Exhaled biomarkers in childhood asthma: old and new approaches. Asthma Res Pract. 2018;4:9.

9. Karrasch S, Linde K, Rucker G, et al. Accuracy of FENO for diagnosing asthma: a systematic review. Thorax. 2017;72: 109-116.

10. Petsky HL, Kew KM, Chang AB. Exhaled nitric oxide levels to guide treatment for children with asthma. Cochrane Database Syst Rev. 2016;11:CD011439.

11. Kotecha S, Clemm H, Halvorsen T, Kotecha SJ. Bronchial hyperresponsiveness in preterm-born subjects: a systematic review and meta-analysis. Pediatr Allergy Immunol. 2018;29:715-725.

12. Kotecha SJ, Edwards MO, Watkins WJ, et al. Effect of preterm birth on later FEV1: a systematic review and meta-analysis. Thorax. 2013;68: 760-766.

13. Kotecha SJ, Edwards MO, Watkins WJ, Lowe J, Henderson AJ, Kotecha S. Effect of bronchodilators on forced expiratory volume in $1 \mathrm{~s}$ in preterm-born participants aged 5 and over: a systematic review. Neonatology. 2015;107: 231-240.

14. Been JV, Lugtenberg MJ, Smets E, et al. Preterm birth and childhood wheezing disorders: a systematic review and meta-analysis. PLoS Med. 2014;11: 1001596.

15. Edwards MO, Kotecha SJ, Lowe J, Richards L, Watkins WJ, Kotecha S. Management of prematurity-Associated wheeze and its association with atopy. PLoS ONE. 2016;11: e0155695.

16. Review Manager (RevMan) [Computer program]. Version 5.3. Copenhagen: The Nordic Cochrane Centre, The Cochrane Collaboration, 2014.

17. Wan X, Wang W, Liu J, Tong T. Estimating the sample mean and standard deviation from the sample size, median, range and/or interquartile range. BMC Med Res Methodol. 2014;14:135.

18. https://training.cochrane.org/resource/revman-calculator

19. Nordlund B, James A, Ebersjo C, Hedlin G, Brostrom EB. Differences and similarities between bronchopulmonary dysplasia and asthma in schoolchildren. Pediatr Pulmonol. 2017;52: 1179-1186.

20. Baraldi E, Bonetto G, Zacchello F, Filippone M. Low exhaled nitric oxide in school-age children with bronchopulmonary dysplasia and airflow limitation. Am J Respir Crit Care Med. 2005;171: 68-72.

21. Kilbride H, Dinakar C, Carver $\mathrm{T}$, et al. Pulmonary function, oxygen consumption, and exhaled nitric oxide measures for extremely low birth weight, heavier preterm, and term children. J Invest Med. 2012;60:239.

22. Morsing E, Gustafsson P, Brodszki J. Lung function in children born after foetal growth restriction and very preterm birth. Acta Paediatrica. Int J Paediatr. 2012;101: 48-54. 
23. Kwinta $\mathrm{P}$, Lis $\mathrm{G}$, Klimek $\mathrm{M}$, et al. The prevalence and risk factors of allergic and respiratory symptoms in a regional cohort of extremely low birth weight children (<1000g). Iltal J Pediatr. 2013;39.

24. Praprotnik M, Gantar IS, Lucovnik M, Avcin T, Krivec U. Respiratory morbidity, lung function and fitness assessment after bronchopulmonary dysplasia. J Perinatol. 2015;35: 1037-1042.

25. Korhonen PH, Suursalmi PH, Kopeli T, et al. Inflammatory activity at school age in very low birth weight bronchopulmonary dysplasia survivors. Pediatr Pulmonol. 2015;50: 683-690.

26. Beckmann J, Bennett K, Bolton C, Marlow N, Hurst J. Respiratory outcomes in young adults following extremely preterm birth. Eur Resp J Conf. 2016;48:PA4167.

27. Cazzato S, Ridolfi L, Bernardi F, Faldella G, Bertelli L. Lung function outcome at school age in very low birth weight children. Pediatr Pulmonol. 2013;48: 830-837.

28. Caskey S, Gough A, Rowan S, et al. Structural and functional lung impairment in adult survivors of bronchopulmonary dysplasia. Ann Am Thorac Soc. 2016;13: 1262-1270.

29. Kaplan E, Bar-Yishay E, Prais D, et al. Encouraging pulmonary outcome for surviving, neurologically intact, extremely premature infants in the postsurfactant era. Chest. 2012;142: 725-733.

30. Vollsaeter M, Clemm HH, Satrell E, et al. Adult respiratory outcomes of extreme preterm birth a regional cohort study. Ann Am Thorac Soc. 2015;12: 313-322.

31. Mieskonen ST, Malmberg LP, Kari MA, et al. Exhaled nitric oxide at school age in prematurely born infants with neonatal chronic lung disease. Pediatr Pulmonol. 2002;33: 347-355.

32. Hafkamp-de Groen E, Sonnenschein-van Der Voort AMM, Mackenbach JP, et al. Socioeconomic and sociodemographic factors associated with asthma related outcomes in early childhood: the generation R study. PLoS ONE. 2013;8:e78266.

33. Holster IL, Sonnenschein-van der Voort AMM, Duijts L, et al. Maternal helicobacter pylori colonization is not associated with asthma symptoms, airway inflammation and airway resistance in their children until the age of 6 years: the generation $r$ study. Gastroenterology. 2013;144:S-5.

34. Rucci E, den Dekker HT, de Jongste JC, et al. Maternal fatty acid levels during pregnancy, childhood lung function and atopic diseases. The Generation R Study. Clin Exp Allerg. 2016;46: 461-471.
35. Pike KC, Inskip HM, Robinson SM, et al. The relationship between maternal adiposity and infant weight gain, and childhood wheeze and atopy. Thorax. 2013;68: 372-379.

36. Vollsaeter M, Skromme K, Satrell E, et al. Children born preterm at the turn of the millennium had better lung function than children born similarly preterm in the early 1990s. PLoS ONE [Electronic Resource]. 10:e0144243.

37. Malmberg LP, Pelkonen AS, Malmstrom K, et al. Very low birth weight and respiratory outcome: association between airway inflammation and hyperresponsiveness. Ann Allerg Asthma Immunol. 2013;111: 96-101.

38. Hennessy EM, Bracewell MA, Wood N, et al. Respiratory health in preschool and school age children following extremely preterm birth. Arch Dis Child. 2008;93: 1037-1043.

39. Narang I, Rosenthal M, Cremonesini D, Silverman M, Bush A. Longitudinal evaluation of airway function 21 years after preterm birth. Am J Respir Crit Care Med. 2008;178: 74-80.

40. Joshi S, Powell T, Watkins WJ, Drayton M, Williams EM, Kotecha S. Exercise-induced bronchoconstriction in school-aged children who had chronic lung disease in infancy. J Pediatr 2013;162:813-818. e811.

41. Kotecha SJ, Watkins WJ, Lowe J, Granell R, Henderson AJ, Kotecha S. Comparison of the associations of early-Life factors on wheezing phenotypes in preterm-Born children and term-Born children. American Journal of Epidemiology 2019; In Press.

\section{SUPPORTING INFORMATION}

Additional supporting information may be found online in the Supporting Information section at the end of the article.

How to cite this article: Course CW, Kotecha S, Kotecha SJ. Fractional exhaled nitric oxide in preterm-born subjects: $\mathrm{A}$ systematic review and meta-analysis. Pediatric Pulmonology. 2019;54:595-601. https://doi.org/10.1002/ppul.24270 